\title{
La generación de conocimiento científico en un marco de responsabilidad social universitaria en la Universidad Nacional del Chaco Austral
}

\author{
Oestmann, Germán \\ germanoestmann@uncaus.edu.ar \\ Universidad Nacional del Chaco Austral \\ Stegagnini, Lucas \\ lucasstegagnini@uncaus.edu.ar \\ Universidad Nacional del Chaco Austral \\ Fogar, Ricardo \\ rfogar@uncaus.edu.ar \\ Universidad Nacional del Chaco Austral \\ Romero, Mara \\ mara@uncaus.edu.ar \\ Universidad Nacional del Chaco Austral \\ ${ }^{2}$ Instituto de Investigaciones en Procesos Avanzados INIPTA-CONICET \\ Fernández, Carina \\ carinafernandez@uncaus.edu.ar \\ Universidad Nacional del Chaco Austral
}

\section{RESUMEN}

La Responsabilidad Social Universitaria (RSU) puede definirse como un modelo de gestión en el que la institución dirige sus esfuerzos a inculcar e implementar un conjunto de principios y valores en cada integrante de la comunidad universitaria, de manera de fomentar un desempeño personal y académico consciente de la necesidad del cuidado del medio ambiente y comprometido con el desarrollo de la sociedad. Bajo esta premisa, a partir de 2019 la Universidad Nacional del Chaco Austral (UNCAUS) ha implementado un programa de RSU, cuyos lineamientos han llevado a diseñar una serie de actividades que permitieron la puesta en marcha de investigaciones interdisciplinarias aplicadas a problemáticas concretas, de modo que investigadores y docentes se encuentren trabajando sobre la misma problemática, en el mismo lugar, desde sus respectivas especialidades, creando así una sinergia de disciplinas desde un enfoque integral. En este artículo se describirán brevemente estas experiencias y los proyectos de investigación resultantes, 
demostrando la posibilidad de generar conocimiento científico en un marco de RSU en la UNCAUS, lo que permitirá orientar la investigación universitaria hacia hallazgos tendientes a resolver problemáticas de la comunidad en la que la institución está inmersa.

Palabras clave: articulación de funciones sustantivas; aprendizaje-servicio; extensión universitaria; ética profesional; conciencia social 


\title{
Scientific knowledge generation under a framework of university social responsibility for at National University of Chaco Austral
}

\begin{abstract}
University Social Responsibility (USR) can be defined as a management model in which the institution directs its efforts to instill and implement a set of principles and values in each member of the university community, in order to promote personal and academic performance committed to the social development and aware of the aware of the importance of the environmental impact. In accordance with this, from 2019 the National University of Chaco Austral (UNCAUS) has implemented a USR program, whose guidelines have led to the design of a set of activities that led to the implementation of two interdisciplinary research applied to specific problems, thus creating a synergy of disciplines from a comprehensive approach. These experiences and the resulting research projects will be briefly described in this article, demonstrating the possibility of generating scientific knowledge within a framework of USR at UNCAUS, which will guide university research towards findings aimed at solving local community problems.
\end{abstract}

Keywords: integration of the substantive functions; service learning; third mission; professional ethics; social conscience

Artículo recibido: 10 Agosto. 2021 Aceptado para publicación: 07. Setiembre. 2021 Correspondencia: carinafernández@uncaus.edu.ar Conflictos de Interés: Ninguna que declarar 


\section{INTRODUCCIÓN}

La Responsabilidad Social Universitaria (RSU) puede definirse como un modelo de gestión en el que la institución dirige sus esfuerzos a inculcar e implementar un conjunto de principios y valores en cada integrante de la comunidad universitaria, de manera de fomentar un desempeño personal y académico consciente de la necesidad del cuidado del medio ambiente y comprometido con el desarrollo de la sociedad (Valleyls 2014). Es así que el enfoque de RSU atraviesa en forma transversal a los cuatro procesos básicos de la universidad (gestión, docencia, investigación y extensión), alineando la misión universitaria con el compromiso social, mediante la interacción de toda la comunidad universitaria con los múltiples actores sociales interesados en el buen desempeño universitario y necesitados de él (Giuffré y Ratto, 2014; Valleys, 2014). En este contexto, desde el año 2000 a la fecha se ha consolidado el concepto de RSU en varios países de Iberoamérica (Martí y Martí-Vilar, 2013; Olarte-Mejía y Ríos-Osorio, 2015; Vallaeys y Álvarez, 2019) al incorporar elementos relevantes para la sociedad relacionados con los impactos sociales, económicos y medioambientales, así como con los aspectos relativos a la ética y la transparencia en la relación de la universidad con las partes interesadas en el quehacer de las instituciones de educación superior a nivel mundial (Gaete, 2015; Larrán y Andrades, 2015; Licandro y Yepes, 2018; Martí-Noguera, Licandro y Gaete, 2018; Vallaeys, 2018).

No obstante, tal como lo expone Valleyls (2014), si bien la finalidad de la RSU está claramente definida, su implementación enfrenta numerosas barreras, desde políticas e ideológicas hasta económicas y estratégicas, siendo entre todas estas quizás la más fuerte la barrera de las mentalidades, ya que es frecuente que actores universitarios defensores de una universidad comprometida con su entorno social, caigan en confusiones y malinterpretaciones conceptuales que hacen muy difícil la promoción de una RSU realmente transformadora. Una de estas malinterpretaciones es el considerar erróneamente a la RSU como una función más dentro de la universidad, separada de las funciones sustantivas, lo que la condena de antemano a no ser nunca una dimensión transversal a todas las acciones institucionales, confundiendo la responsabilidad social con una función específica de la universidad, lo que implica el inconveniente que las demás funciones no ejerciten el concepto de responsabilidad social, acorralando a la RSU hacia el ámbito específico de los proyectos sociales voluntarios con comunidades vulnerables externas a la 
universidad, y reduciéndola al hecho de cumplir con "buenas acciones" hacia fuera de la universidad (Vallaeys, 2018). Algo similar sucede con la investigación científica, donde es frecuente que autoridades e investigadores consideren a la RSU totalmente apartada de la generación de conocimiento científico, cuando en realidad sucede todo lo contario, ya que la investigación en el ámbito universitario puede ser de gran utilidad para el desarrollo social, tecnológico, económico y cultural, desde una perspectiva ética capaz de develar desde los aportes científicos el comportamiento del objeto o sujeto de estudio, bajo todas las formas posibles para transformarlo en soluciones que contribuyan a superar problemas reales (Menjivar, 2020).

Con la premisa de involucrar a la comunidad universitaria con los ciudadanos de la región y generar plena conciencia de la realidad provincial y de sus vulnerabilidades y necesidades, a partir de 2019 la Universidad Nacional del Chaco Austral (UNCAUS) ha implementado un programa de RSU, cuyos lineamientos han llevado a diseñar una serie de actividades que permitieron proponer investigaciones interdisciplinarias aplicadas a problemáticas concretas, de modo que investigadores y docentes se encuentren trabajando sobre la misma problemática, desde sus respectivas especialidades, creando una sinergia de disciplinas desde un enfoque integral. En este artículo se describirán brevemente estas experiencias y los proyectos de investigación resultantes, demostrando la posibilidad de generar conocimiento científico en un marco de RSU en la UNCAUS, lo que permitirá orientar la investigación universitaria hacia hallazgos tendientes a resolver problemáticas de la comunidad en la que la institución está inmersa.

\section{EXPERIENCIAS ENMARCADAS EN EL PROGRAMA DE RSU DE LA} UNCAUS

La Universidad Nacional del Chaco Austral se encuentra en la localidad de Presidencia Roque Sáenz Peña, en la provincia del Chaco, al norte de la Argentina. Su oferta académica incluye 17 carreras presenciales, algunas de las cuales permiten el empleo de estrategias educativas como el aprendizaje-servicio, lo que permite integrar el servicio a la comunidad con la enseñanza y la reflexión (Martínez-Usarralde, Gil-Salom y Macías-Mendoza, 2019) mediante prácticas que conducen al logro de habilidades y actitudes sociales en los estudiantes, las cuales son puestas en práctica en la vida diaria, promoviendo así la formación de ciudadanos críticos, activos y responsables (Francisco y Moliner, 2010; Chiva-Bartoll y Gil-Gómez, 2018). 
En el marco del programa de RSU, se diagramaron en forma articulada actividades de docencia, extensión e investigación, las que se realizaron en el periodo julio-diciembre de 2019 con dos grupos conformados por docentes, alumnos e investigadores pertenecientes a las carreras de Medicina, Licenciatura en Nutrición e Ingeniería en Alimentos. Empleando la metodología de enseñanza aprendizaje-servicio, cada uno de los grupos realizó tareas de extensión, promoviendo uno de ellos hábitos saludables de alimentación y beneficios de la donación de leche materna el otro. Estas temáticas se abordaron atendiendo a dos problemáticas de nuestra comunidad: por un lado, los problemas de malnutrición en poblaciones vulnerables y, por otro, la falta de conocimientos respecto de la donación de leche materna, lo que representa un inconveniente considerando que el hospital de nuestra ciudad registra un número cada vez mayor de nacimientos prematuros y el Banco de Leche Humana (BLH) más cercano se encuentra a $160 \mathrm{~km}$ de nuestra ciudad. Además de las actividades de promoción, se realizaron encuestas a los ciudadanos participantes, relevando uno de los grupos el valor nutricional de los alimentos consumidos frecuentemente, y el otro indagando sobre la opinión de los asistentes respecto de la creación de un BLH en la ciudad (Martin, Verniere, Bondar, Fogar, Romero, 2019). Los resultados de estas encuestas mostraron, por un lado, un consumo de alimentos fuente de hierro preocupantemente bajo, principalmente debido al costo de los mismos, y, por otra, la conveniencia de encontrar formas de prolongar la vida útil de la leche materna donada.

En base a estos resultados se pusieron en marcha dos proyectos de investigación, ambos actualmente en etapa de ejecución, denominado el primero de ellos "Ingredientes funcionales a base de derivados de sangre de ganado bovino", que surgió a partir del bajo consumo de alimentos fuente de hierro registrado en el trabajo de campo previo. Considerando que la carencia de hierro es la principal causa de anemia y que ésta es una problemática tanto en nuestra comunidad como en cualquier parte del mundo, especialmente niños en edad escolar y embarazadas, se considera oportuno utilizar la sangre de ganado vacuno proveniente de mataderos, la cual es un residuo tradicional de la industria frigorífica. Por lo tanto, el objetivo del proyecto es desarrollar ingredientes ricos en hierro de alta absorción, para emplearlos en la elaboración de preparaciones culinarias frecuentemente consumidas, tales como guisados, sopas y panificados, lo que implica diversas formas de incorporación, ya sea como geles o como ingrediente en polvo. Hasta el momento se han realizado pruebas de caracterización fisicoquímica de los ingredientes 
propuestos (Fernández, Skopiuk, Fogar, Marri y Judis, 2018; Fernández, Skopiuk, Fogar, Romero, y Doval, 2018), se han realizado encuestas evaluando su potencial aceptabilidad (Fernández, Ambroggio y Trinajstic, 2018) y se ha ensayado su incorporación en pan y bizcochos (Morales, Riernersman, Romero y Doval, 2019; Campos, Michaluk, Doval y Fernández, 2019). Restan realizar ensayos de aceptabilidad de preparaciones culinarias elaboradas con estos ingredientes, tras lo cual se prevé la firma de convenios entre el gobierno provincial, la universidad y organizaciones gubernamentales y no gubernamentales de la localidad para que en un trabajo conjunto estos ingredientes sean accesibles a las poblaciones de interés, como una forma de contribuir a la prevención de la anemia por carencia de hierro. Además, al usar la sangre de matadero como fuente de hierro de alta absorción se contribuye al cuidado del medio ambiente, ya que se transforma un residuo tradicional en una materia prima.

El segundo proyecto puesto en marcha se denominada "Conservación de leche materna mediante deshidratación", cuyo objetivo es encontrar la forma óptima conservar leche materna donada al someterla a un tratamiento de deshidratación, de manera de dotar al BLM a crearse en las dependencias de la Unidad Médica Educativa (UME) de la universidad con un producto de mayor vida útil. Esto redundaría en beneficios para los casos de prematuros cuyas madres no pueden darles de mamar y no cuentan con donantes en el momento (lo cual es muy frecuente), considerando que la leche materna donada solo puede conservarse en su estado original por un periodo no mayor a 12 horas (Martin y col., 2019). Hasta el momento se han realizado análisis fisicoquímicos a la leche donada por madres asistentes a consulta ginecológica y/o pediátrica en la UME (Barriales, Sturla, Fogar, Romero, 2019; Martin, Radovancich, Polischuk, Fogar, Romero, 2019) pero el contexto de pandemia no ha permitido obtener el volumen de leche donada necesario para pasar a la etapa de deshidratación por liofilización y posterior evaluación de las condiciones de almacenamiento, actividades que se tienen previstas para el año en curso. Por otra parte, ya que desde 2011 nuestro país cuenta con la Comisión Técnica Asesora en Bancos de Leche Materna (Ministerio de Salud, 2010) a partir de la cual se crearon BLM en diferentes puntos del país, se cuenta con el aval del Gobernador de la provincia para la creación/implementación de un BLM en la Unidad Médica Educativa de la UNCAUS, el que servirá como unidad de apoyo clínico al Servicio de Neonatología que funciona en dicha unidad y cubrirá parte de la demanda provincial de leche materna . 


\section{CONSIDERACIONES FINALES}

Dado que las universidades son las responsables de formar a la gran mayoría de los profesionales de un país, su primera respuesta ante la sociedad debe ser educar jóvenes con alto nivel de conocimientos, alto nivel de conciencia de los problemas sociales y alta capacidad de reflexionar sobre el impacto de sus acciones tanto en el ambiente como en la sociedad. Por ello, la planificación y ejecución articulada de actividades de docencia, extensión e investigación enmarcadas en un programa de RSU como las aquí descritas representan una oportunidad de generar conocimiento científico que ofrezca soluciones reales a la comunidad en la que se encuentra inserta la universidad. De este modo será posible que estudiantes, docentes e investigadores aprendan y se involucren en acciones de cuidado del medio ambiente, siguiendo una conducta de respeto hacia los demás, lo que contribuirá a la reforzar los hábitos éticos de convivencia, que poco a poco irán permeando primero a las familias, y posteriormente a la sociedad en su conjunto.

\section{LISTA DE REFERENCIAS}

Barriales, V. N., Sturla, M. A., Fogar, R. A. y Romero, M. C. (2019). Caracterización de lípidos presentes en leche materna mediante cromatografía en capa fina. Reunión de Difusión de la Labor Docente, Científica, Tecnológica y de Extensión. 1a ed. Presidencia Roque Sáenz Peña: UNCAUS. Argentina.

Campos, O., Michaluk, A. G., Doval, M. M. Y Fernández, C. L. (2019). Caracterización de panes con alto contenido de hierro hémico obtenidos a partir de fracciones deshidratadas de sangre bovina. Memorias XXI Congreso Latinoamericano Y Del Caribe De Ciencia Y Tecnología De Alimentos \& XVII Congreso Argentino De Ciencia Y Tecnología De Alimentos. Universidad Católica Argentina. Sede Puerto Madero. Buenos Aires. Argentina.

Chiva-Bartoll, Ó. y Gil-Gómez, J. (eds.) (2018). Aprendizaje-Servicio Universitario. Modelos de intervención e investigación en la formación inicial docente, Barcelona, Octaedro.

Fernández, C. L., Ambroggio, V. M. y Trinajstic, M. A. (2018). Alimentación complementaria de lactantes y preparaciones culinarias potencialmente fortificables con hierro hemínico en centros de salud de Pcia. Roque Sáenz Peña. Reunión de Difusión de la Labor Docente, Científica, Tecnológica y de Extensión. 1a ed. Presidencia Roque Sáenz Peña: UNCAUS. Argentina. 
Fernández, C., Skopiuk, E., Fogar, R., Marri, D. y Judis, M. A. (2018). Estabilidad de emulsiones $\mathrm{O} / \mathrm{W}$ elaboradas con mezclas de fracciones de sangre de bovino como agente emulsionante. Reunión de Difusión de la Labor Docente, Científica, Tecnológica y de Extensión. 1a ed. - Presidencia Roque Sáenz Peña: UNCAUS. Argentina.

Fernández, C., Skopiuk, E., Fogar, R., Romero, M. y Doval, M. (2018). Obtención de hidrogeles proteicos para su uso como potencial sustituto de grasa animal en productos cárnicos. Reunión de Difusión de la Labor Docente, Científica, Tecnológica y de Extensión. 1a ed. - Presidencia Roque Sáenz Peña: UNCAUS. Argentina.

Francisco, A. y Moliner, L. (2010). El Aprendizaje Servicio en la Universidad: una estrategia en la formación de ciudadanía crítica. Revista Electrónica de Formación de Profesorado, 13(4), 69-77.

Gaete, R. (2015). La responsabilidad social universitaria desde la perspectiva de las partes interesadas: un estudio de caso. Revista Electrónica Actualidades Investigativas en Educación, 15(1), 1-29. DOI: dx.doi.org/10.15517/aie.v15i1.17729

Giuffré, L. y Ratto, S. E. (2014). A New Paradigm in Higher Education: University Social Responsibility (USR). Journal of Education \& Human Development, 3(1), 231-238.

Larrán, M. y Andrades, F. (2015). Análisis de la responsabilidad social universitaria desde diferentes enfoques teóricos. Revista Iberoamericana de Educación Superior, 6(15), 91-107.

Licandro, Ó. y Yepes, S. (2018). La educación superior conceptualizada como bien común: El desafío propuesto por UNESCO. Revista Digital de Investigación en Docencia Universitaria, 12(1), 6-33. doi: http://dx.doi.org/10.19083/ridu.12.716

Martí, J. J. y Martí-Vilar, M. (2013). Una década de responsabilidad social en Iberoamérica. Revista Española del Tercer Sector, 25, 145-162.

Martí-Noguera, J. J., Licandro, Ó. y Gaete, R. (2018). La responsabilidad social de la educación superior como bien común. Concepto y desafíos. Revista de la Educación Superior, 47(186), 1-22.

Martin, C. E., Radovancich, V. M.; Polischuk, T., Fogar, R. A. y Romero, Mara C. (2019). Reunión de Difusión de la Labor Docente, Científica, Tecnológica y de Extensión. 1a ed. - Presidencia Roque Sáenz Peña: UNCAUS. Argentina. 
Martin, C. E., Verniere, M. L., Bondar, S. A., Fogar, R. A. y Romero, M. C. (2019). Conocimientos sobre bancos de leche humana en madres que asisten a Centros De Atención Primaria De Salud (CAPS) de Sáenz Peña. Reunión de Difusión de la Labor Docente, Científica, Tecnológica y de Extensión. 1a ed. - Presidencia Roque Sáenz Peña: UNCAUS. Argentina.

Martin, C. E., Verniere, M. L., Sang, M., Fernández, C. L., Fogar, R., A. y Romero, M. C. (2019). Vinculación entre lactancia materna y donación de leche humana como eje central en la alimentación del recién nacido. Congreso Latinoamericano. El Rol Social De La Educación Superior En América Latina: Desafíos, Escenarios y Prácticas Innovadoras. Universidad Nacional del Chaco Austral. Presidencia Roque Sáenz Peña, Chaco.

Martínez-Usarralde, M. J., Gil-Salom, D. y Macías-Mendoza, D. (2019). Revisión sistemática de Responsabilidad Social Universitaria y Aprendizaje servicio. Análisis para su institucionalización. Revista Mexicana de Investigación Educativa, 24(80), 149-172.

Menjivar, W. (2020). La responsabilidad social universitaria: un enfoque para contribuir al desarrollo desde la Educación Superior. Ciencia, Cultura y Sociedad, 6(1), 5-7.

Ministerio de Salud. (2010). Comisión Técnica Asesora en Bancos de Leche Materna. Recuperado de: http://servicios.infoleg.gob.ar/infolegInternet/anexos/175000179999/176806/norma.htm

Morales, L. H., Riernersman, C. N., Romero, A. M., Doval, M. M. y Fernández, C. L. (2019). Estudio del valor nutricional y propiedades fisicoquímicas de bizcochos con oleogel de aceite de girasol alto oleico como sustituto de grasa bovina. Memorias XXI Congreso Latinoamericano Y Del Caribe De Ciencia Y Tecnología De Alimentos \& XVII Congreso Argentino De Ciencia Y Tecnología De Alimentos. Universidad Católica Argentina. Sede Puerto Madero. Buenos Aires. Argentina.

Olarte-Mejía, D. y Ríos-Osorio, L. (2015). Enfoques y estrategias de responsabilidad social implementadas en Instituciones de Educación Superior. Una revisión sistemática de la literatura científica de los últimos 10 años. Revista de la Educación Superior, 44(175), 19-40. 
Vallaeys F. (2104). La responsabilidad social universitaria: un nuevo modelo universitario contra la mercantilización. Revista Iberoamericana de Educación Superior, 12(5), 105-107.

Vallaeys, F. (2018). Las diez falacias de la Responsabilidad Social Universitaria. Revista Digital de Investigación en Docencia Universitaria, 12(1), 34-58. doi: http://dx.doi.org/10.19083/ridu.12.716

Vallaeys, F. y Álvarez, J. (2019). Hacia una definición latinoamericana de responsabilidad social universitaria. aproximación a las preferencias conceptuales de los universitarios. Revista Educación XX1, 22(1), 93-116. doi: https://doi.org/10.5944/educxx1.19442 